\title{
The Modeling and Simulations of Low Noise Floor Solar Radio Receiver
}

\author{
Wang $\mathrm{Hao}^{1}$, Du Qingfu, ${ }^{*}, 1,2$, Song Yong ${ }^{1}$ and Chen $\mathrm{Yao}^{2}$ \\ ${ }^{I}$ School of Mechanical, Electrical \&Information Engineering, Shandong University, Weihai 264209, P.R. China \\ ${ }^{2}$ Shandong Provincial Key Laboratory of Optical Astronomy and Solar-Terrestrial Environment, and Institute of Space \\ Sciences, Shandong University, Weihai 264209, P.R. China
}

\begin{abstract}
In the field of solar radio, the high performance solar radio receiver which has high sensitivity, large dynamic range is necessary to observe the radio storm fine structure. The low noise floor solar radio receiver can meet the observation request. By the theory analysis of the radio storm receiver's noise floor and combining with the characteristic of solar radio storm, the paper submits a method based on adding low noise amplifier(LNA) before receiver and decreasing the frequency resolution bandwidth(RBW) to lower the solar receiver noise floor. The paper gives the verification of the design method by means of the modeling and simulations. And the verified results comply with the design target.
\end{abstract}

Keywords: Dynamic range, frequency resolution bandwidth (RBW), low noise amplifier (LNA), low noise floor, sensitivity, solar radio receiver.

\section{INTRODUCTION}

The phenomenon that radiation intensity of radio wavebands increase suddenly when the solar burst is called solar radio storm. Studying the physical information carried by solar radio storm such as the process of solar burst, shack wave generation and evolution, electron acceleration and the radiation mechanism not only contribute to understand and diagnose the physical process of related and basic plasma, but also know important information like triggering and energy release with transformation mechanism, mass movement and so on in the process of explosion [1,2]. Besides, studying solar radio which is only celestial body radio radiation that can be measured in high resolution and precision also contribute to understand the similar radiation process of celestial body like space stars. So the observation and further study of solar radio have important science study value. The solar radio receiver is an important equipment to receive and study the solar radio storm. The receiver's performances can influence the originality of solar radio storm signals directly. The solar radio storm signals have some characteristics such as wide frequency range, many weak signals, high dynamic range. So the receiver to observe the solar radio must have some good performances such as high sensitivity, high dynamic range and low noise floor. Meanwhile, the noise floor of receiver can affect the sensitivity and dynamic range directly [3]. It is definite to improve the receiver's noise floor by the theory analysis of receiver noise floor. The modeling and simulations can verify the design method.

*Address correspondence to this author at the School of Mechanical, Electrical \&Information Engineering, Shandong University at Weihai, weihai, Shandong, 264209, P.R. China; Tel: +86 06315688066; Fax: +86 06315688338; E-mail: dqf@sdu.edu.cn

\section{THE THEORY BASIS OF SOLAR RADIO RECEIVER NOISE FLOOR}

The noise floor of receiver is also called background noise. The receiver internal noise is called thermal noise [4], is produced by the electronic thermal motion related to environment temperature and signal bandwidth. Its definition is thermal noise power level which is intercepted by receiver's passband when the temperature is $290 \mathrm{~K}\left(17^{\circ} \mathrm{C}\right)$. The thermal noise power level is also called noise floor of receiver is a basic parameter to calculate noise power.

$P_{n}=10 \lg (K T B)+N F=-174+10 \lg (B)+N F$

where,

K---the Boltzmann constant $(1.38 \cdot 10-23 \mathrm{~J} / \mathrm{K})$;

T---the Kelvin temperature;

B---the signal bandwidth of receiver;

NF [5]---the noise figure of receiver;

$10 \lg (\mathrm{KTB})$---the receiver's Gaussian white noise(produced by electronic thermal motion in system );

$-174 \mathrm{dBm} / \mathrm{Hz}$---the noise power spectral density at normal temperature(electronic system's thermal noise power per $\mathrm{Hz}$ at normal temperature).

$$
\begin{aligned}
& n_{0}=10 \lg \left(\frac{K T B}{B}\right) \\
& =10 \lg (K T)=10 \lg \left(1.38 \cdot 10^{-23} \cdot 290 \cdot 10^{-3}\right) \\
& =-174 \mathrm{dBm} / \mathrm{Hz}
\end{aligned}
$$

The noise floor of receiver mainly influences the sensitivity and dynamic range. The lower the noise floor of 
the receiver, the higher the sensitivity and the larger the dynamic range of the receiver. The sensitivity and dynamic range are two very important performances in observation of solar radio storm. But these two parameters are influenced by noise floor of the receiver directly.

The sensitivity of receiver [6] is defined as minimum signal level which is received at the input port under the certain condition of reference measured channels' throughput meeting the demand.

If the minimum useful signal is wanted to extract from system noise floor, then the minimum useful signal power must be greater than or equal to the system noise floor power.

$S=P_{n}+S N R$

where,

S---the sensitivity of receiver;

$\mathrm{P}_{\mathrm{n}^{--}}$the noise floor of receivers.

In general, the sensitivity of receiver is greater SNR about $7 \mathrm{~dB}$ than the noise floor power. According of equation (4), the lower the noise floor, the higher the sensitivity of receiver. So that the receiver can receive the less power solar radio storm signals. In order to improve the sensitivity of receiver, the noise floor of receiver must be reduced that could influence the sensitivity directly.

In general, the difference value size of maximum signal and minimum signal in one measurement is called the dynamic range of the receiver. The dynamic range of receiver is mainly influenced by the electronic components such as amplifiers and mixers used in receiver and the noise floor of receiver. The larger the noise floor of receiver, the smaller the dynamic range of receiver. Then, if the dynamic range is small, the number of signals observed is less. So that it is difficult to analyze the solar radio storm fine structure detailedly.

By the equation (1), the noise floor of receiver is composed of natural noise (the noise intercepted by receiver passband) and the noise figure of receiver system. Then for improving the sensitivity and dynamic range of receiver, reducing the noise floor is a good way from the natural noise (the noise intercepted by receiver passband) and the noise figure of receiver system two parts.

\section{THE DESIGN SCHEME OF LOW NOISE FLOOR RECEIVER}

Based on the receiver noise floor of the theoretical analysis, combining with the characteristics of the solar radio burst such as wide range of signal frequency, many weak signals, large dynamic range, the designed receiver must have the advantages of high sensitivity, large dynamic range. But noise floor influence these two parts. The lower the noise floor, the higher the sensitivity and the larger the dynamic range. For improving the sensitivity and dynamic range of receiver, reducing the noise floor is a good way done from the natural noise (the noise intercepted by receiver passband) and the noise figure of receiver system two parts. So it is worth to think about reducing natural noise (the noise intercepted by receiver passband) and the noise figure of receiver system to lower the noise floor of receiver in the design process of the receiver.

\subsection{Reduce the Frequency Resolution Bandwidth}

The bandwidth of signals can influence the receiver natural noise (the noise intercepted by receiver passband). Under the condition of fixed noise figure of receiver, the smaller the bandwidth (BW), the lower the noise floor of receiver shown in equation(1). In observing the solar radio burst, the frequency resolution (RBW) $[7,8]$ is an important concept. In practical application, $\mathrm{RBW}$ is the minimum frequency interval which can distinguish two different frequency signals [9]. Under the certain conditions of a fixed noise power meaning with fixed bandwidth $\mathrm{B}$ and fixed environment temperature, the less RBW means the whole noise power divided to $\mathrm{B} / \mathrm{RBW}$ filters, so the noise floor will reduce. But the amplitude of these signals is fixed. Based on equation (1), the noise floor of receiver is

$P_{n}=-174+10 \lg (R B W)+N F$

If the NF of receiver is $30 \mathrm{~dB}$. Different $\mathrm{RBW}$ gets different noise floor in theory in $290 \mathrm{~K}\left(17{ }^{\circ} \mathrm{C}\right)$ shown in Table 1.

Table 1. Different RBW and different noise floor in theory in $290 K\left(17^{\circ} \mathrm{C}\right)$.

\begin{tabular}{|c|c|c|c|c|c|}
\hline $\mathbf{R B W}(\mathrm{Hz})$ & $\mathbf{1 0 0 0 0}$ & $\mathbf{1 0 0 0}$ & $\mathbf{1 0 0}$ & $\mathbf{1 0}$ & $\mathbf{1}$ \\
\hline \hline Noise Floor(dBm) & -104 & -114 & -124 & -134 & -144 \\
\hline
\end{tabular}

Referring to Table 1, it shows that the smaller the RBW, the lower the noise floor of receiver under the certain condition of fixed NF. So the sensitivity and dynamic of receiver can be improved well with the RBW smaller. Thus it is very convenient to observe the solar radio storm fine structure. So it is wise to reduce the RBW as much as possible when designing the solar radio receiver. The less $\mathrm{RBW}$ can reduce the noise floor of receiver, but means that analyzing the whole bandwidth spends more time. If so, some short duration signals are lost possible.

\subsection{Adding Low Noise Amplifier at Receiver Frontend}

Adding the low noise amplifier at receiver frontend can reduce the NF and the noise floor of receiver. Fig. (1):

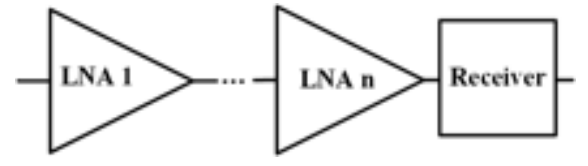

Fig. (1). $n$ Low noise amplifier and receiver cascade.

According to the NF calculation formula of cascade structure:

$\mathrm{F}_{\mathrm{n}}=\mathrm{F}_{1}+\frac{\mathrm{F}_{2}-1}{\mathrm{G}_{1}}+\frac{\mathrm{F}_{3}-1}{\mathrm{G}_{1} \mathrm{G}_{2}}+\frac{\mathrm{F}_{4}-1}{\mathrm{G}_{1} \mathrm{G}_{2} \mathrm{G}_{3}}+\bullet \bullet \frac{\mathrm{F}_{n}-1}{\mathrm{G}_{1} \ldots \mathrm{G}_{n}}$

$N F=10 \lg \left(F_{n}\right)$ 
According of Equation (4) and Equation (5), the NF of cascade structure are mainly determined by the first component. In general, the NF of low noise amplifier is very small. Cascading a low noise amplifier before receiver can reduce the NF of whole system. But due to some devices within receiver (such as mixer, ADC, etc) linearity and input range limit, the dynamic range of whole receiver system may be smaller after adding the low noise amplifier.

The dynamic range of receiver must be considered and coordinate with each other combining with practical application when using this way to improve the noise floor of receiver.

\section{THE MODELING AND MULATIONS OF LOW NOISE FLOOR RECEIVER}

\subsection{The Scheme of Improving Noise Floor by Changing RBW}

The paper simulated the scheme of changing RBW to improve the receiver's noise floor by the MATLAB modeling. The flow chart of simulation is shown in Fig. (2). First, set the parameters of the initial value such as $\mathrm{N}$ for FFT points, RBW, span for frequency band, $f_{c}$ for the center frequency of band-pass filter. If $\mathrm{f}_{\mathrm{c}}<$ span, so signals with noise are inputted to window function to reduce the spectrum leakage. At the same time, the signals after window do $\mathrm{N}$ points FFT arithmetic. Then, calculate the power average of the FFT results. The average power corresponds to the frequency $f_{c}$. Then to change the center frequency of bandpass filter according the formula $\mathrm{f}_{\mathrm{c}}=\mathrm{f}_{\mathrm{c}}+\mathrm{RBW}$. The cycle continues until the $f_{c}>$ span. Finally, get the power spectrum whose frequency and power are one to one correspondence.

The simulation modeling input signals are three same amplitude and different frequency signals mixing with white noise. The frequencies of three signals are $50 \mathrm{kHz}, 52 \mathrm{kHz}$, $80 \mathrm{kHz}$ respectively. In Fig. (3), it is shown that the noise floor is about $-116 \mathrm{dBm}$ when the RBW is $10 \mathrm{kHz}$. It is difficult to distinguish the $50 \mathrm{kHz}$ signal between $52 \mathrm{kHz}$ signal. In Fig. (4), it is shown that the noise floor is about $125 \mathrm{dBm}$ when the RBW is $1 \mathrm{kHz}$. It is easy to distinguish the $50 \mathrm{kHz}$ between $52 \mathrm{kHz}$ signal.

Comparing Fig. (3) with Fig. (4), the noise floor is smaller about $10 \mathrm{~dB}$ obviously when the RBW reduce the ten times. It accords with theoretical equation (3). The more signals will be observed if the noise floor is lower which means higher sensitivity.

\subsection{Cascading LNA to improving noise floor}

It is also obvious to reduce the noise floor of receiver when the low noise amplifier (LNA) is cascaded at the front of receiver. So the Aglient N9320B spectrum analyzer in laboratory was used to experiment. In Fig. (5), it is shown that the system noise floor was $-104 \mathrm{dBm}$ and the observed signals were environmental radio signals when the LNA was not cascaded at the front of spectrum analyzer. In Fig. (6), it is shown that the system noise floor was about $-120 \mathrm{dBm}$ and the number of observed radio signals increased obviously when the LNA was cascaded at the front of spectrum analyzer. Meanwhile, many small radio signals were observed.

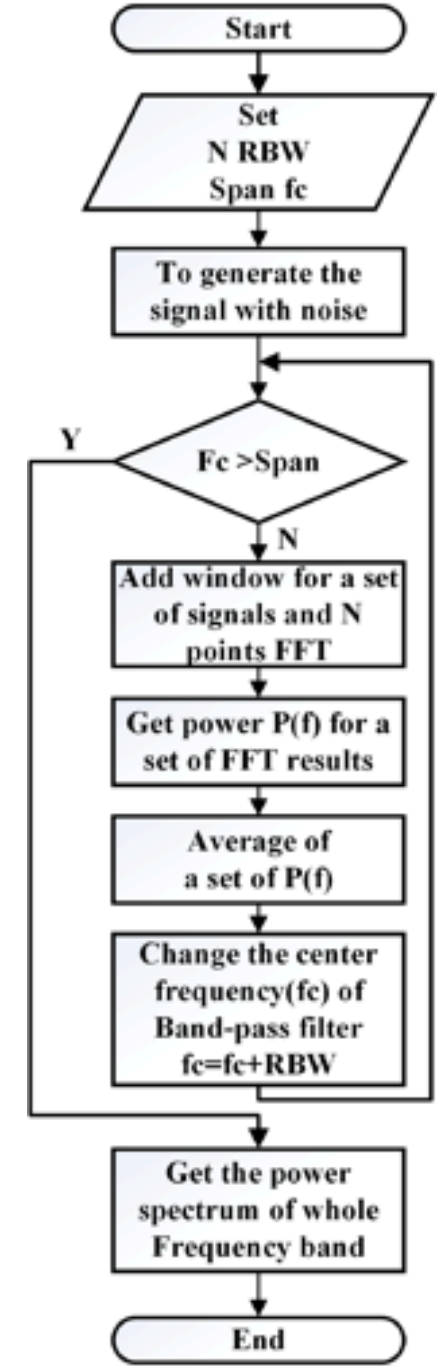

Fig. (2). Modeling flow chart.

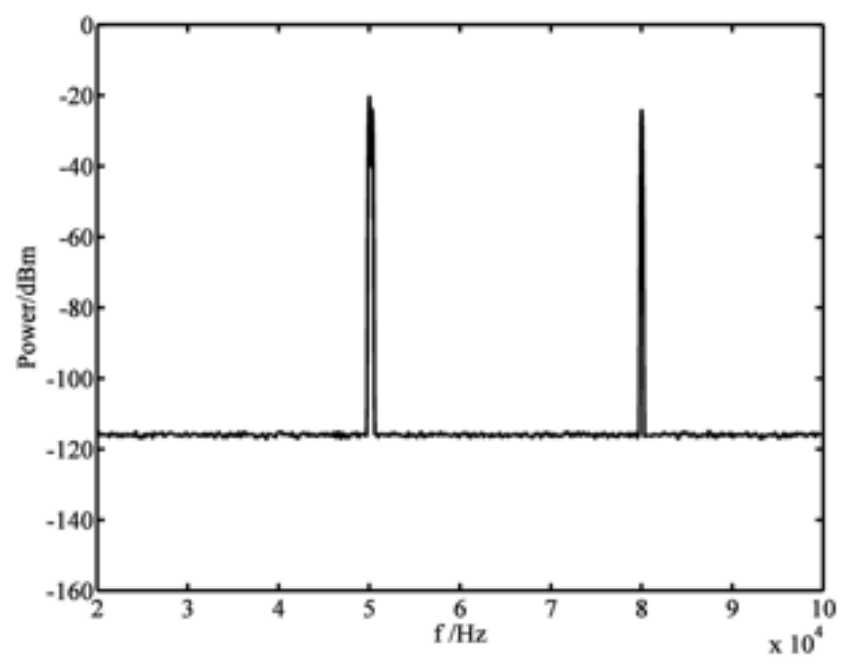

Fig. (3). The simulation of $10 \mathrm{kHz} \mathrm{RBW}$.

Comparing Fig. (5) with Fig. (6), the noise floor reduced about the gain $(16 \mathrm{~dB}$ in this experiment) of LNA when the LNA was cascaded at the front of spectrum analyzer. The more small signals will be observed if the noise floor is 
lower which means higher sensitivity. In other words, the signals submerged in noise could be detected. Thus, this way can provide much data in more detail for researchers to study the solar radio storm fine structure. But some amplified signals are greater than the maximum of LNA so that the dynamic range may reduce.

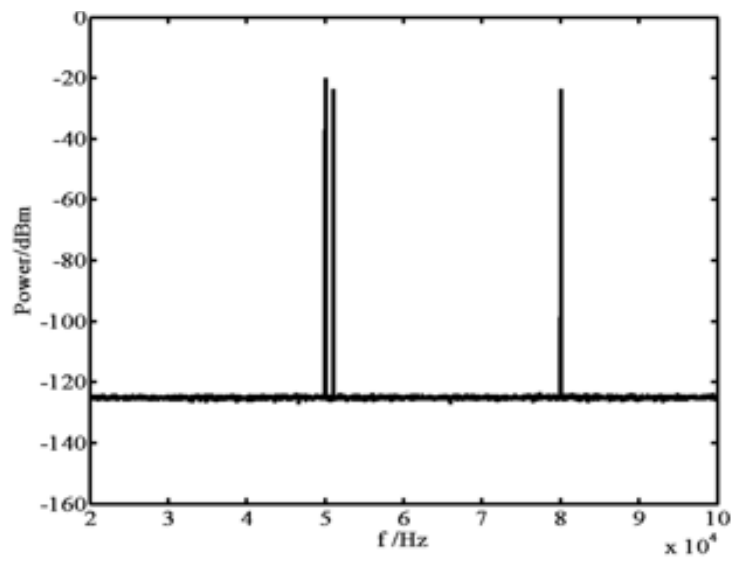

Fig. (4). The simulation of $1 \mathrm{kHz}$ RBW.

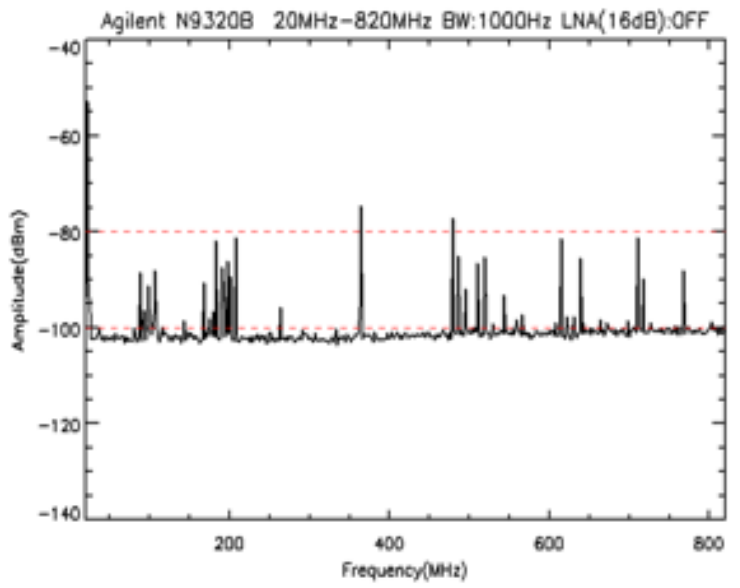

Fig. (5). Not add $16 \mathrm{~dB}$ LNA to Observe 20M-820MHz signals.

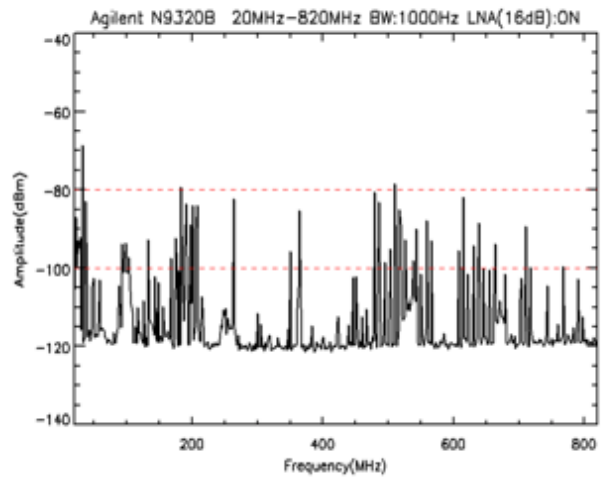

\section{CONCLUSION}

It is effective to be lower the noise floor of receiver and then improve the sensitivity and the dynamic well when add the LNA at receiver frontend and reduce the RBW known from the theoretical analysis and the modeling simulations. But due to some devices within receiver (such as mixer, $\mathrm{ADC}$, etc) linearity and input range limit, the dynamic range may reduce when the LNA is added at receiver frontend. At the same time, the less RBW means that analyzing the whole bandwidth spends more time. So it is very necessary to consider the performance requirements of whole system and coordinate with each other to achieve the balance of best performance when design the low noise receiver by adding LNA and reducing RBW.

\section{CONFLICT OF INTEREST}

The authors confirm that this article content has no conflict of interest.

\section{ACKNOWLEDGEMENTS}

This Project was supported by The National Natural Science Foundation of China (61473174), The Specialized Research Fund for the Doctoral Program of Higher Education (20130131130006).

\section{REFERENCES}

[1] Guanjun Gao, Juan Lin and Min Wang, "Solar meter and decimeter type II, type III radio bursts and fine structure observation and research progress," Progress in Astronomy, vol.30, no.1, pp. 34-36, Feb.2011.

[2] Xiangliang Kong, "The acceleration and radiation research of the shock waveelectron insolar bursts", PhD dissertation, Shandong University, Jinan: Shandong University,2014.

[3] Xingwen Zeng, Naijian Liu and Jian Chen, The Principle and Analysis of High Frequency Circuit, CA:Xi'an, 2010, pp.10-62.

[4] Jinzhan Gao, Weak Signal Detection, CA:Beijing, 2004, pp.42-59.

[5] Shibai Tong. Fundamentals of Analog Electronic Technology, CA: Beijing,2006, pp.100-292.

[6] Yun Li, "Correction of Spectrum Analyzer Sensitivity Analysis and Small Signal Measurement," Radio Engineering, vol.42, no.1, pp.62-63, Jan. 2012.

[7] Peiqing Cheng, Digital Signal Processing Course, CA:Beijing, 2008, pp.44-194.

[8] Chengling Zhao, Chao Zhang. "By Over Sampling Technique and FFT Algorithm to Improve the Dynamic Range of Measurement," Science \& Technology Information, vol.17, pp.88-89,2008.

[9] Chinese product center of Keysight technologies, "Spectrum analysis

foundation,’Nov.2012;http://blog.sina.com.cn/s/blog_8c5f60cc010 $11 \mathrm{f0w} \cdot \mathrm{html}$.

Fig. (6). Add 16dB LNA to observe 20M-820MHz signals. 